\title{
Successes of Modelling Parkinson Disease in Drosophila
}

\author{
Brian E. Staveley \\ Memorial University of Newfoundland \\ Canada
}

\section{Introduction}

Over one hundred years of innovative experimentation with the "common fruit fly" or Drosophila melanogaster has placed this remarkable organism at the forefront of contemporary biological research. Whether we consider the implications of modern genetic technologies and comprehensive genomic research, or we are interested in leading-edge aspects of molecular and cellular biology or complex developmental biology systems, or our studies range from the pin-point accuracy of proteomics to the large-scale questions of population biology, research with fruit flies has made very significant contributions to our understanding of the basic and complex functions of life. Although research into a wide range of biological questions has benefited greatly from experimentation with Drosophila, it never fails to surprise how often an approach that uses this model organism is undervalued or ignored. Any experimental system can be and should be criticized; however the opportunity to explore the biological basis of disease should never be missed. One shining example of this point is our understanding of Parkinson disease which has expanded through and continues to benefit from basic research into the biology of Drosophila, a model organism whose genome has been so thoroughly understood as to make it indispensable for medical research. These recent advances provide significant support for the use of Drosophila melanogaster models in the study of the biological basis of many human diseases and disorders.

Parkinson disease is the most common movement disorder and the second most common neurodegenerative disorder. Most apparent to even the most casual of observers is the fact that patients with Parkinson disease present with symptoms that are related to locomotion and motor control. These symptoms include resting tremor, slowness of movement, rigidity and postural instability. As the common underlying source that gives rise to these movement difficulties, Parkinson disease is most often associated with and distinguished by the degeneration of neurons, especially the dopamine-producing, or dopaminergic, neurons in the substantia nigra of the midbrain and the subsequent loss of dopamine (Dauer \& Przedborski, 2003). Associated with these degenerating neurons is the appearance in many cases of the disease of large aggregates of proteins that are often referred to as the Lewy bodies. Often ignored are the additional non-motor symptoms, where non-dopaminergic neurons including olfactory and brain stem neurons, can frequently deteriorate before the dopaminergic neurons show signs of loss (Braak et al., 2003). Additional Parkinson disease symptoms are common and comprise a number of behavioural symptoms that include 
dementia, depression, anxiety and difficulties with sleep, plus non-behavioural symptoms such as the development of muscular and skeletal anomalies and skin lesions (Simuni \& Sethi, 2008). Given the importance and the complexity of this disease, the application of a multifaceted, interdisciplinary approach to understanding the biological basis of Parkinson disease, including the modelling of the disease in well-known genetically versatile organisms, cannot be stressed enough.

The inherited or familial forms of Parkinson disease are, for the most part, indistinguishable in nature and severity from the sporadic forms of the disease (Hardy et al., 2009). As of only a few years ago, Parkinson disease was believed to be completely sporadic in nature, yet the identification of the inherited forms of the disease along with subsequent characterization of the causative genetic defects has revolutionized this area of research. It is now known that the familial cases comprise approximately 10 to $15 \%$ of the cases and arise from mutations in several identified genes with new loci associated with Parkinson disease being routinely discovered. Of the loci identified early in this process, mutations in the alpha-synuclein gene (designated as both PARK1/PARK4) (Polymeropoulos et al., 1997; Singleton et al., 2003) and Leucine-rich repeat kinase 2 or LRRK2 (identified as PARK8) (Paisan-Ruiz et al., 2004; Zimprich et al., 2004) cause autosomal dominant or "gain-of-function" forms of the disease. Mutations in the parkin gene (designated as PARK2)(Kitada et al., 1998), PTEN-induced kinase 1 or Pink1 (PARK6)(Valente et al., 2004), and Dj-1 (PARK7) (Bonifati et al., 2003) are associated with autosomal recessive or "loss-of-function" forms of Parkinson disease. With the identification of the underlying genetic contributions to, at the very least, a sizable proportion of the incidents of Parkinson disease, it has become possible to apply the principles of disease modelling in genetically tractable animal systems to the study of this disease.

To demonstrate the great utility of the application of research into Drosophila melanogaster in the modelling of Parkinson disease, I will describe some of the most exciting recent advances in this field. To begin, a brief description of the wealth of genetic and transgenic approaches that are most commonly used to model aspects of human disease in Drosophila will be provided as an introduction to the organism. The first model of Parkinson disease, one based upon the toxic expression of the human alpha-synuclein gene, the first gene identified as a genetic cause of Parkinson disease, will be discussed. This model offered the opportunities to study a wide range of biological contributions to Parkinson disease including aspects of protein structural stability, oxidative stresses and other disease genes. Investigation of Lrrk/LRRK2 in flies has revealed roles in dopamine distribution, protein synthesis and cell death in another model of a dominant form of Parkinson disease. Then, the processes of modelling Parkinson disease through examination of the loss-of-function of the Drosophila homologues of the parkin and Pink1 genes, both responsible for recessive forms of Parkinson disease will be detailed. Of a very significant nature, this area of research has lead to the fundamental understanding the activity of these gene products at the mitochondria. Furthermore, recent studies have lead to the proposal of a mechanism that outlines the normal role of parkin and Pink1 in mitochondrial dynamics. It is very likely that the loss of this mechanism leads to failure of the cell's ability to clear damaged mitochondria and ultimately results in the degeneration of these cells and, subsequently, the disease state. Finally, continued study of Drosophila models of Parkinson disease is clearly well positioned to contribute a great deal to the future of research into the basis of this disease. 


\section{The Drosophila approach to model human disease}

Drosophila melanogaster has been extensively studied and a wealth of genetic, genomic, cellular and developmental knowledge and reagents are readily available. Drosophila are inexpensive to propagate and can produce a large number of genetically homogenous progeny. Flies display surprisingly intricate behaviours and have complicated brain and nervous systems. For many purposes, the fly provides a well-characterized system that is relatively easy to manipulate but complex enough to be relevant to the development of human disease models.

Often, once a gene has been implicated in a given disease, a bioinformatic search of genomic sequences can readily identify a potential homologue or orthologue from among the genes that comprise the well-characterized genome of Drosophila melanogaster as well as the genomes of a number of other Drosophila species for comparison. Application of reverse genetics can lead the production of both loss-of-function and gain-of-function phenotypes that may recapitulate symptoms of a given disease. Loss-of-function can be achieved either through the creation of mutations that lower or abolish gene activity or through the directed expression of an interfering RNAi transgene. A gain of function phenotype can be generated by the directed expression of a gene to where there is normally low or no expression or by inducing elevated levels of expression far above the norm. As a very well studied system, the Drosophila's pre-existing loss-of-function mutations, as well as transposon-bearing lines that can be employed to direct the expression, are available through stock centres and individual research laboratories from around the world. Due to the genomics projects, it is easy to access cDNA and genomic clones along with various vectors for generation of a variety of transgenic animals.

\subsection{Drosophila genetics: genes, mutants and transgenics}

In most cases, genes that have been identified as playing a role in human disease have counterparts in the genome of Drosophila melanogaster. Through the analysis of pre-existing mutants, the application of genetic screens to generate novel loss-of-function mutants and the implementation of "interfering RNA" or RNAi technology to reduce or eliminate gene activity can mimic the effect of recessively inherited diseases. For dominantly inherited disease, some pre-existing dominantly inherited alleles may exist in the stock centre collections. However, hypermorphic gain-of-function phenotypes can be generated by the directed overexpression of a transgene introduced into the fly genome through germ-line transformation. In addition, neomorphic and antimorphic gain-of-function phenotypes may be produced by in vitro construction and transformation of Drosophila transgenes that replicate molecular defects that have been determined to cause disease in people. Alternatively, a transgene that can express a variant form of a human gene can be fashioned and transformed. This approach has been applied to study the function and the potential contribution of genes to a number of diseases, including Parkinson disease. This approach can be instrumental in providing insight into the function of an identified gene involved in a human disease when the function is difficult to determine.

To produce the gain-of-function or the RNAi loss-of-function phenotypes described above, the Gal4/UAS system has been widely employed for the ectopic expression of specific genes in Drosophila (Brand \& Perrimon 1993). For the most part, a gene of interest is cloned within a $\mathrm{P}$ element transposon-bearing plasmid containing several copies of the DNA-binding target of the yeast transcription factor GAL4 designated as the 'Upstream Activating 
Sequence' (UAS) along with a selectable marker to readily track the presence of the transgene. As there is no Drosophila transcription factor that acts though binding of this sequence, in the absence of GAL4 these fusion transgenes are mostly inactive. Once established, individuals bearing a responsive transgene can be mated to specific lines that express Gal4 in any one or combination of expression patterns. Many choices of expression are available including ubiquitous, pan-neural, dopaminergic neuron-specific, early or late in eye development, inducible by heat shock, and many more. When the Gal4 transgene and the UAS target gene are combined in the offspring of the controlled breeding experiments, the gene of interest is subject to control of expression with regard to level, timing and tissue specificity. Analyses of these progeny provide the opportunity to approach a wide range of fundamental biological investigations including the detailed modelling of human disease.

When dealing with living systems, and in particular when carrying out complex manipulation of a model organism, careful evaluation and consideration of the techniques employed are necessary. With this in mind, it is important to note that a very limited number of Gal4 transgenes had been demonstrated to lead to cell death: first in the neuronrich compound eye (Kramer \& Staveley, 2003) and later in the nervous system (Rezaval et al., 2007). This suggests that the use of the Gal4/UAS system requires some caution because there may be a compounding Gal4-effect in some experiments when interpreting experimental observations. As always, control experiments must be subjected to proper evaluation and scrutiny. Nevertheless, the Gal4/UAS ectopic gene expression system in Drosophila is an extremely powerful tool and is one of the reasons that modelling human disease in flies is such an attractive pursuit.

\subsection{Drosophila dopaminergic neurons}

For a model system to functionally approach a condition as complex as Parkinson disease, changes to specific tissues that result in recapitulation of phenotypes that resemble symptoms of the disease are key. The Drosophila adult brain has been characterized to contain clusters of dopaminergic neurons (Nassel \& Elekes, 1992). The feeding of rotenone, the complex I inhibitor that initiates degeneration of dopaminergic neurons in mammals, can cause the loss of these clusters of dopaminergic neurons in flies (Coulom \& Birman, 2004). This demonstrates that there is a susceptibility of dopamine-producing neurons to toxins that is conserved between mammals and flies. As described below, altering the expression of selected genes in these dopaminergic neurons has resulted in loss of the neurons coupled with an age-dependent loss of locomotor abilities. The basic similarities between the genetically manipulated Drosophila models and Parkinson disease patients, given that the loss of the dopamine-producing neurons and the subsequent change in behaviour occurs over time, suggests that significant aspects of the disease may be well modelled in flies.

\section{The alpha-synuclein-dependent Drosophila model of Parkinson disease}

Once the genetic basis of a familial form of Parkinson disease was identified (the alphasynuclein gene or PARK1 and, later, PARK4 as well) and the molecular basis of the defect determined (as specific changes to the protein), exploring the function or dysfunction of this gene was greatly aided by study in the Drosophila system. 


\subsection{The alpha-synuclein gene (PARK1) models Parkinson disease in Drosophila}

Although instances are rare, mutations that result in substitution of amino acid residues within the human alpha-synuclein protein, specifically A30P, A53T and E46K, produce a dominant autosomally inherited version of Parkinson disease (PARK1; Polymeropoulos et al. 1997; Kruger et al. 1998; Zarranz et al. 2004). An early onset familial version of Parkinson disease results when a duplication of the region bearing this gene produces an increase in gene copy number (PARK4; Singleton et al. 2003). Although abundant in Lewy bodies, the precise physiological function of alpha-synuclein is uncertain. Because two of the original designated PARK loci (PARK1 and PARK4) have mapped to the alpha-synuclein gene, the native role and consequences of dysfunction appear complex and deserve to undergo extensive evaluation.

It is of extreme importance to the modelling of Parkinson disease in Drosophila that the first and most exploited Drosophila model of Parkinson disease depends upon the Gal4/UAS system for the ectopic expression of various forms of the human alpha-synuclein gene (Feany \& Bender, 2000). Expression of human wild-type and Parkinson disease-causing mutant forms of alpha-synuclein in Drosophila results in the loss of the dopamine-producing neurons. The loss of neurons is coupled with the loss of the ability to climb over time in adult flies. As well, the development of Lewy body-like cytoplasmic inclusions and degeneration of the retina occurs with the expression of A30P, A53T and wild-type versions of alpha-synuclein using different Gal4 drivers. The observed phenotypic end-points produced by the disease-associated forms of the alpha-synuclein protein appear to display some differences and may mirror aspects of the toxicity that lead to various sub-types of Parkinson disease. To be clear, bioinformatic analysis of the genome of Drosophila melanogaster has not identified genes that encode any member of the synuclein family of proteins, including alpha synuclein. However, the recapitulation of phenotypes in Drosophila caused by the toxicity of alpha-synuclein gene product that somewhat mimic the consequences of Parkinson disease certainly seems to validate such study in this model organism. As with Parkinson disease patients, Drosophila models of Parkinson disease involve multiple defects, the cellular basis that provides insight into the pathogenesis of Parkinson disease.

\subsection{Controversial detection of neurodegeneration in the model}

Briefly, visualization of the dopaminergic neurons in Drosophila brains or brain sections has been accomplished through two main methods: detection of the tyrosine hydroxylase enzyme via immunohistochemistry, or detection of a green fluorescent protein or GFP reporter gene placed under the control of the tyrosine hydroxylase-Gal4 transcription factor. For the most part, the determination of the specific death of dopaminergic neurons over time in response to the expression of alpha-synuclein has been demonstrated reproducibly since development of the alpha-synuclein-induced Drosophila model of Parkinson disease (Feany \& Bender, 2000; Auluck et al., 2002; Cooper et al., 2006; Wassef et al., 2007; Botella et al., 2008; Trinh et al., 2008). Not all studies could detect dopaminergic neurodegeneration using anti-tyrosine hydroxylase immunostaining (Pesah et al., 2005) or detection of transgenic GFP (Whitworth et al. 2006). Clearly differences in the approach or methodology used to measure loss of dopaminergic neurons can influence the sensitivity of the assay (Auluck et al., 2005). In addition, as a definite decrease in the strength of the nuclear GFP signal has been observed the dopaminergic neurons of ageing flies that express alpha-synuclein, (Botella 
et al. 2008) a significant proportion of the differences could have easily been overlooked. In primary cultures of neurons cultivated from Drosophila that express alpha-synuclein, an in vitro model for Parkinson disease that shows great potential, the observed decrease in detection of GFP in these cultured neurons has been strongly associated with the early stages of apoptosis and signs of neurodegeneration (Park et al., 2007). Although this has been a contentious issue, the loss of immunological staining of the tyrosine hydroxylase enzyme, or detection of the tyrosine hydroxylase-responsive GFP reporter, seems to be very well correlated to neuronal dysfunction and degeneration.

This controversy highlights one great advantage of the study of Parkinson disease in an organism that presents complex phenotypes that reflect the consequences of the disease in humans. Of great importance, experiments where the loss of neurons is correlated to the loss of locomotor ability over time have provided sufficient comparison. For example, when oxidative stress is prevented, the loss of locomotor activity and the loss of dopaminergic neurons are diminished together in flies expressing alpha-synuclein (Pendleton et al., 2002, Yang et al., 2003, Wassef et al., 2007, Botella et al., 2008). As such, the loss of climbing ability seems to be a meaningful and modifiable phenotype that allows for the detection and validation of subtle influences.

\subsection{Mechanisms to prevent alpha-synuclein-dependent toxicity}

To address the possibility that chaperone activity may actively counteract protein toxicity, elevated expression of Heat Shock Protein 70 (HSP70) was demonstrated to toxicity of asynuclein expression although aggregates were found (Auluck et al., 2002). Reduced chaperone activity contributed to increased loss of neurons resulting from expression of alpha-synuclein. Providing transgenic flies with geldanamycin, an inducer of chaperone activity, added to the food source contributed to survival of alpha-synuclein-expressing neurons (Auluck et al., 2005). The phosphorylation of alpha-synuclein at residue Serine residue-129, a modification often found in brains from Parkinson disease patients, apparently leads to toxicity (Fujiwara et al., 2002). In Drosophila melanogaster, study of the consequences of in vivo alteration of this site of phosphorylation suggests that this aminoacid residue is responsible for keeping the alpha-synuclein protein in a soluble form instead of in a state of aggregation (Chen \& Feany, 2005). Prevention of phosphorylation at this site results in aggregation and reduced toxicity. This observation supports the hypothesis that the soluble form of the protein has a much greater potential for toxicity than does the nonphosphorylated form. Taken all together, this suggests that the process of aggregation acts as a protective cellular mechanism that works to neutralize the toxic forms of the alphasynuclein protein.

While oxidative stress seems to contribute to Parkinson disease, a relationship to the mechanism behind alpha-synuclein toxicity is unclear. The degeneration of neuronal phenotypes induced by expression of mutant forms of alpha-synuclein is enhanced growth under conditions of hyperoxia while the elevated expression of the oxygen free radial scavenger superoxide dismutase suppresses the neuronal degeneration and the loss of locomotor activity over the lifespan of alpha-synuclein-expressing flies (Botella et al., 2008). To support the role of oxidative insult in the disease process, the alpha-synuclein-induced phenotypes are suppressed by other known antioxidants. These include the overexpression of methionine sulfoxide reductase and the supplementation of the Drosophila growth medium with S-methyl-L-cysteine (Wassef et al., 2007), and the induction of glutathione 
synthesis or glutathione conjugation activity (Trinh et al., 2008). As the toxicity of alphasynuclein is sensitive to oxidative stress, manipulation of antioxidants may make a significant contribution to modify these effects.

One of the great advantages of employing the Drosophila model is the ability to combine and evaluate various components identified to contribute to Parkinson disease in an animal model organism. The first example of combining gene products that are known to cause inherited forms of Parkinson disease was the demonstration that the overexpression of parkin can act to counteract the toxic effects of both wild type and mutant forms of alphasynuclein to restore climbing ability and to prevent degeneration of the retina when coexpressed in the eye (Haywood \& Staveley, 2004; 2006). Similarly, the directed expression of Pink1, an upstream activator of parkin, acts to restore locomotor abilities and prevent subtle developmental defects in the eye (Todd \& Staveley, 2008). This approach demonstrates that the directed expression of some of the recessive Parkinson genes can act to balance defects caused by a dominantly inherited Parkinson disease gene.

\section{The LRRK2/Lrrk (PARK8) models of Parkinson disease in Drosophila}

Leucine-rich repeat kinase 2 or LRRK2 (first identified as PARK8) causes an autosomal dominant or "gain-of-function" form of Parkinson disease (Paisan-Ruiz et al., 2004; Zimprich et al., 2004). Dysfunction of LRRK2 has been mapped to several amino-acid substitutions in the protein and is very prominent among sporadic and inherited forms of Parkinson disease. The LRRK2 gene encodes a very large protein with a leucine-rich repeat (LRR) domain, a kinase domain, a RASlike GTPase domain and WD-40 domain and is very similar to an orthologous gene LRRK1.

There is a single homologue, Lrrk, in Drosophila melanogaster. Perhaps due to difficulties in the detection of neurodegeneration, as discussed above, there has been some confusion with studies of Lrrk/LRRK2 in flies. In one case, Lrrk mutant flies display impaired locomotive activity and a reduction in the immunostaining of tyrosine hydroxylase in dopaminergic neurons (Lee et al., 2007). While the dopaminergic neurons display abnormal morphologies, the absolute number of the neurons appears to be unchanged although they may be degenerating slowly. Under other circumstances, Lrrk mutants seemed relatively normal which lead to the claim that Lrrk is not required for the survival of dopaminergic neurons (Wang et al., 2008). Directed expression of wild type of Lrrk did not lead to detectable degeneration of dopaminergic neurons (Lee et al., 2007). However, expression of mutant forms of both human (G2019S) and Drosophila (I2020T) did lead to documented loss of dopaminergic neurons (Imai et al., 2008; Liu et al., 2008). Nevertheless, the few studies of Lrrk/LRRK2 in flies have revealed a great deal that may influence our understanding of Parkinson disease.

The Lrrk mutants undergo lipid peroxidation and mutant flies containing the carboxyterminal kinase domain truncated Lrrk transgene are sensitive to hydrogen peroxide (Wang et al., 2008). However, Lrrk mutants seem to be reasonably resistant to the oxidative stresses presented by paraquat and hydrogen peroxide (Imai et al., 2008). The relationship between Lrrk/LRRK2 and oxidative stress is unclear for now.

Lrrk/LRRK2 has been demonstrated to be involved in the negative regulation of normal levels of dopamine. The over expression of select mutants of Lrrk, but not wild-type Lrrk, causes a severe reduction in the dopamine levels of the brain (Imai et al., 2008). Treatment with I -DOPA causes improvement in movement not survival of dopaminergic neurons (Liu 
et al., 2008). Conversely, dopamine content is highly elevated in Lrrk mutants, as changes in dopamine levels must not be due to survival neurons but due to either defects in metabolism or processing and handling.

The eukaryotic initiation factor 4E (eIF4E)-binding protein, a major controller of protein synthesis and as such a key regulator of responses to cellular stress is phosphorylated by the Lrrk/LRRK2 kinase (Imai et al., 2008). This strongly suggests that the survival of dopaminergic neurons is compromised by pathogenic forms of Lrrk/LRRK2 through the deregulation or mis-regulation of protein translation. The Lrrk and LRRK2 proteins have been shown to phosphorylate and activate the transcription factor foxo (Kanao et al., 2010). This suggests that downstream targets of foxo, such as hid in flies (and Bim in humans), act to activate the apoptotic machinery to cause the neurodegeneration in Lrrk/LRRK2 models of PD. These research findings demonstrate a very meaningful connection between the control of protein synthesis, activation of cell death programs and the development of Parkinson disease.

\section{The parkin/Pink1-dependent Drosophila models of Parkinson disease}

\subsection{The consequences of parkin and Pink1 loss in Drosophila}

The loss-of-function in the parkin gene (PARK2), which encodes a highly conserved ubiquitin E3 ligase, is responsible for a rare autosomal recessive subtype of Parkinson disease. The Drosophila parkin gene is highly expressed in the Drosophila central nervous system (Horowitz et al. 2001; Bae et al. 2003). In parkin mutants, the dopaminergic neurons degenerate (Greene et al., 2003; Cha et al., 2005; Whitworth et al., 2005; Wang et al., 2007). In flies that that overexpress some mutant forms of parkin, dopaminergic neurons degeneration occurs (Sang et al., 2007). Although viable, parkin mutants present with a reduction in life-span, locomotor defects and extensive degeneration of muscle fibres, the latter is clearly associated with mitochondrial deterioration (Greene et al., 2005). The male parkin mutants are sterile due to failure of mitochondrial activities during spermatogenesis (Riparbelli and Callaini, 2007). Reduction of neuronal-specific staining (either GFP or TH) and/or cell death has been reported in these parkin mutants (Greene et al., 2003; Cha et al., 2005; Whitworth et al., 2005; Wang et al. 2007). The localization of this protein to the mitochondria (Darios et al., 2003) coupled with the consequences of parkin loss is a strong indication that it protects this organelle.

Mutations in the PTEN-induced kinase 1 (Pink1 or PARK6) gene are a common cause of autosomal recessive Parkinson disease (Valente et al., 2004). In flies, the Pink1 gene, like parkin, is highly expressed in adult heads and testes (Park et al. 2006). The Pink1 serinethreonine kinase, along with a kinase domain, contains a mitochondrial-targeting signal (Clark et al., 2006). A decrease in the levels of dopamine with age plus a somewhat limited loss of dopaminergic neurons was found in the Pink1 mutants along with the presence of abnormal mitochondria in the surviving dopaminergic neurons. When the function of Pink1 was inhibited through the directed expression of an RNAi transgene, loss of dopaminergic neurons as well as the age-dependent degeneration of ommatidia was observed (Wang et al. 2006; Yang et al. 2006). The potential for a functional association with the mitochondria and the similarity in the consequences of dysfunction clearly suggest a shared role for these two proteins.

\subsection{The parkin and Pink1 proteins act in one pathway}

Although not identical, the flies that have lost Pink1 function share a number of defects found in the parkin mutants including shortened lifespan, apoptotic muscle degeneration, 
male sterility, defects in mitochondrial morphology and disruption of locomotor abilities. These mitochondria are lost with age from the dopaminergic neurons of Pink1 mutants. While, for the most part, double Pink1-parkin mutants show the same phenotypes as either of the single mutants, the overexpression of parkin is able to rescue the mitochondrial defects found in Pink1, whereas Pink1 overexpression does not rescue the parkin phenotypes. The parkin and Pink1 proteins have been reported to interact physically in at least some contexts (Kim et al., 2008; Xiong et al., 2009). This, at least in part, indicates that the Pink1 and parkin proteins function in the same pathway with Pink1 functioning upstream of parkin activity. This Pink1/parkin pathway is necessary for the integrity of dopaminergic neurons, because the loss of neurons due to loss of Pink1 function could be rescued by additional expression of parkin (Clark et al., 2006; Park et al., 2006). The contribution of the study of the relationship between parkin and Pink1 to our understanding of mitochondrial pathology in Parkinson disease highlights the utility of Drosophila to model Parkinson disease.

Similar to parkin and Pink1, mutations in the Dj-1 gene cause autosomal recessive forms of Parkinson disease (Bonifati et al., 2003) and it has been suggested that they will become another potential component of this pathway. Drosophila melanogaster has two homologues of $\mathrm{Dj}-1$ and is viable when both are deleted or silenced by RNAi transgenes (Menzies et al., 2005; Meulener et al., 2005; 2006). The loss of Dj-1 activities leads to increased sensitivity to oxidative stress when exposed to paraquat or rotenone. However, the overexpression of $D j$ 1 does not rescue the Pink1 mutant phenotypes (Yang et al., 2006). The possibility exists that Dj-1 may act much further upstream or through a parallel mechanism. However, at this time the relationship in unclear and Dj-1 may or may not influence the Pink1/parkin pathway.

\subsection{The parkin and Pink1 proteins co-operate at the mitochondria}

Mitochondria undergo fission and fusion to change shape and share components (Chen \& Chan, 2009). The fusion of mitochondria requires fusion of both the inner and outer mitochondrial membranes. The control of outer membrane fusion requires the activity of the protein mitofusin, and the inner membrane fusion requires the product of the Optic atrophy-1 gene. Mitochondrial fission is promoted by the recruitment of dynamin-related protein 1 (Drp1) to the mitochondria, and this recruitment requires the activity of Fis1, a mitochondrial outer membrane protein. The regulation of this process is essential to the maintenance of a healthy cell.

To focus upon the mitochondria, in the dopaminergic neurons and the adult flight muscles of parkin mutants, the mitochondria are swollen with fragmented cristae (Greene et al., 2003; Pesah et al., 2004). A similar phenomenon is observed in flies that have lost the function of Pink1 (Clark et al., 2006; Park et al., 2006; Yang et al., 2006). During Drosophila spermatogenesis, the spermatid's mitochondria aggregate and fuse to produce the nebenkern, a structure composed of two entangled strings of fused mitochondria. During subsequent elongation, the nebenkern disentangles to yield two fused structures that are maintained throughout the process of spermatogenesis. Whether due to reduced mitochondrial fission or excess fusion, in parkin and Pink1 mutants only one mitochondrial fusion product has been detected (Deng et al., 2008). These mitochondrial defects, along with locomotion defects, flight muscle degeneration, cell death and diminishment of dopamine levels in heads, are suppressed both by the directed expression of the pro-fission genes $d r p 1$ or fis 1 and by decreasing levels of the pro-fusion genes mitofusin or opa1 (Deng et al., 2008; Park et al., 2009; Poole et al., 2008; Yang et al., 2008). In a Pink1 mutant background, 
a reduction in the gene copy number of $d r p 1$, as seen with mutant heterozygotes, causes lethality (Deng et al., 2008; Poole et al., 2008). This clearly suggests that parkin and Pink1 must act to promote mitochondrial fission. It is extremely important to point out that the phenotypes that arise from the loss of parkin or Pink1 are very different from the loss of $d r p 1$ (Deng et al., 2008). This is a strong indication that the Pink1-parkin pathway acts to regulate mitochondrial fission machinery.

\subsection{The regulation of Mitophagy by parkin and Pink1 proteins}

The process of mitophagy is a recently described specialized mitochondrial-specific version of autophagy (Goldman et al., 2010). In this procedure, the mitochondria undergo engulfment by autophagosomes and are degraded. This essential mechanism is absolutely dependent upon the dynamics of the continual fission and fusion of the mitochondria that alter the size and shape of the organelle and allow the exchange of components. With the failure of mitophagy, the quality of cellular respiration is severely diminished as is illustrated through the accumulation of oxidized proteins. Through the regulation of the fission/fusion dynamics of the cellular mitochondria, the Pink1 and parkin collaborate to contribute to this process.

First of all, although the parkin E3 ubiquitin ligase can target toxic proteins for proteasomal degradation, as the loss of parkin results in the accumulation of toxic proteins and overexpression can suppress toxicity of potential targets, other cellular processes can be regulated by ubiquitination by parkin (Geisler et al., 2009). With this in mind, a mechanism has been proposed that outlines the potential roles of the parkin and Pink1 proteins in the regulation of mitochondrial dynamics, changes in which can lead to alterations in the process of mitophagy (Geisler et al., 2009; Narendra et al., 2009; Vives-Bauza et al., 2010; Ziviani et al., 2010). The recruitment of parkin from the cytoplasm to the mitochondria depends on the activity of the Pink1 kinase. Pink1 is localized to the outer mitochondrial membrane through a well-conserved mitochondrial targeting signal peptide located near the amino-terminus of the protein (Zhou et al., 2008). This arrangement leaves the carboxyterminal kinase-containing remainder of the protein exposed to the cytoplasm. It is proposed that under normal conditions, the tethered Pink1 protein is cleaved in a constitutive way to release the portion of Pink1 that contains the kinase activity into the cytoplasm (Narendra et al., 2009; Vives-Bauza et al., 2010; Ziviani et al., 2010). As a result, under standard conditions, Pink1 activity at the mitochondrial membrane is maintained at a steady but low level.

However, when stressed mitochondria undergo a critical amount of damage, the routine degradation of the Pink1 kinase is discontinued (Narendra et al., 2009; Vives-Bauza et al., 2010; Ziviani et al., 2010). This leaves intact and active versions of Pink1 to accumulate at the mitochondrial membrane in response to the termination of Pink1 inactivation. The initiation and maintenance of the accumulation of Pink1 may depend upon a signal generated when the mitochondria are not able to maintain membrane potential. Although the Rhomboid-7 protease is a candidate enzyme that can cleave Pink1 (Whitworth et al., 2008), this protease may not be the one responsible for this activity in response to mitochondria signalling for destruction. In the first major step of this process, the result is the differential identification of damaged mitochondria through the build up of active Pink1 activity bound and secured to the outer mitochondrial membrane. According to this mechanism, the next step in the regulation of mitophagy depends upon the recruitment of the parkin E3 ubiquitin ligase 
though Pink1 activity to the outer mitochondrial membrane (Narendra et al., 2009; VivesBauza et al., 2010; Ziviani et al., 2010). Recruitment of parkin to the mitochondrial membrane depends upon the localization of the Pink1 kinase to the mitochondria. An alternative method of mitochondrial targeting of Pink1 also recruits parkin (Narendra et al., 2009). Finally, the presence of the parkin ligase at the mitochondria results in the ubiquitination and subsequent degradation of the fusion-promoting outer mitochondrial membrane protein mitofusin (Ziviani et al., 2010). The mitofusin protein has been shown to accumulate with the loss of Pink1 and parkin gene functions. If this proposal holds true, the initiation of mitophagy may very well depend upon signaling through the ubiquitination of mitofusin. In turn, whether mitofusin is targeted for degradation or modified to a form that no longer contributes to the process, this situation leads to the prevention of the fusion of the outer mitochondrial membrane. Although there is no evidence that fission is directly influenced, the subsequent failure of damaged mitochondria to be isolated would likely result from their loss of the ability to undergo proper segregation.

In summary, when mitochondria accumulate sufficient damage, the Pink1 protein becomes stabilized at the mitochondria. This acts to recruit parkin to the damaged mitochondria that in turn causes degradation or modification of mitofusin to promote mitochondrial fission and mitophagy to remove these damaged mitochondria. In Pink1/parkin mediated Parkinson disease, the damaged mitochondria are not cleared as efficiently to result in cellular damage.

\section{The future of drosophila models of Parkinson disease}

Drosophila is proving to have great advantages in the genetic and cell biological study of Parkinson disease. As more genes are found to be associated with Parkinson disease, further applications of reverse genetics should lead to greater understanding of the disease. The Drosophila phenotypes offer many diverse clues that may benefit from greater scrutiny. As well as future screens that modify the more obvious phenotypes such as male sterility, muscle degeneration, abnormal wing positioning, locomotion defects as well as mitochondrial defects in multiple tissues in young adults, these can be examined/scored for suppression or enhancement without having to carry out aging studies that can span months. Studies of candidate genes that may modify the activities of genes that mediate familial Parkinson disease are rigorous, but allow for rapid and straightforward means to deduce mechanisms. Given that mitochondria defects accumulate during normal aging, identification of multiple means to activate mitophagy may have applications to many aspects of aging. Drosophila provides an indispensable and unique opportunity to contribute to the Parkinson disease field.

\section{Conclusion}

To summarize, the recent expansion of research interest in the well understood laboratory organism Drosophila melanogaster to provide highly informative models of Parkinson disease demonstrates some of the great advantages that this system has to offer. The first model of Parkinson disease, one based on the toxic expression of the human alpha-synuclein gene, has allowed a great deal of investigation into various biological factors, such as protein folding, oxidative stress and protein detoxification, that may contribute to the disease. Studies of 
Lrrk/LRRK2 have revealed roles for the management of dopamine handling, the control of protein synthesis and the initiation of programmed cell death in models of Parkinson disease. Further modelling of Parkinson disease through careful evaluation of parkin and Pink1 loss-of-function in Drosophila has revealed that these two genes contribute to a pathway where the parkin E3 ubiquitin ligase is under the regulatory control of the Pink1 protein kinase. In flies, the relationship between parkin and Pink1 has been extended from the neurons to other tissues including the mitochondrial-rich flight muscle and the male gametes. This has led to investigation of the products of these highly conserved genes in the processes that promote mitochondrial survival. The routine participation of parkin and Pink1 in mitochondrial activities reveals that the loss of the fusion/fission dynamic can result in the failure of the cells to adequately deal with mitochondria that present a burden to the health of the cell. Future study of Parkinson disease in Drosophila models will continue to reveal much about the basis of this disease. As the past century has made clear, over and over again, investigations that apply our collective knowledge of the genetics and biology of Drosophila melanogaster to explore the fundamentals of disease, such as Parkinson disease, are very difficult to ignore.

\section{Acknowledgments}

I wish to thank the Natural Sciences and Engineering Research Council of Canada (NSERC) Discovery Grants, NSERC Research Tool Instruments Grants, and Parkinson Society Canada Friedman Pilot Project Grants for funding my research programs. In a special acknowledgement, I wish to thank the family of Jerry Friedman for supporting Parkinson disease research in Canada. I wish to thank the School of Graduate Studies at Memorial University of Newfoundland and the NSERC post-graduate scholarship and NSERC undergraduate scholarship programs for funding the students that have studied aspects of Parkinson disease in Drosophila in my laboratory. I thank the talented technical and the patient administrative support staff within the Department of Biology, the Faculty of Science and the Office of Research at Memorial University of Newfoundland. I thank Annika F.M. Haywood, Amy M. Todd, Githure (Peter) M'Angale, Lisa Baker (Saunders), Gillian Sheppard, Sharleen Hoffe, Michael Nightingale, Jo-Anna Clark, Kevin Mitchell, Greg Dale, Meghan O'Leary, Kate Bassett, Heather Stone, Kimberley Chafe, David Lipsett and Jamie M. Kramer for participation in modelling Parkinson disease in Drosophila melanogaster in my laboratory.

Finally, as the recent progress in this field has been extensive, I must offer my sincere apologies to those colleagues whom I have failed to include in this discussion. There are many research groups that have made and continue to make extremely significant contributions to the study of Parkinson disease through the study of the Drosophila model system.

\section{References}

Auluck, P.K.; Chan, H.Y., Trojanowski, J.Q., Lee, V.M. \& Bonini, N.M. (2002). Chaperone suppression of alpha-synuclein toxicity in a Drosophila model for Parkinson's disease. Science, 295, pp. 865-8. 
Auluck, P.K.; Meulener, M.C. \& Bonini, N.M. (2005). Mechanisms of suppression of \{alpha\}synuclein neurotoxicity by geldanamycin in Drosophila. J Biol Chem, 280, pp. 28738.

Bae, Y.J.; Park, K.S. \& Kang, S.J. (2003). Genomic organization and expression of parkin in Drosophila melanogaster. Exp Mol Med, 35, pp. 393-402.

Bonifati, V.; Rizzu, P., van Baren, M.J., Schaap, O., Breedveld, G.J., Krieger, E., Dekker, M.C., Squitieri, F., Ibanez, P., Joosse, M., van Dongen, J.W., Vanacore, N., van Swieten, J.C., Brice, A., Meco, G., van Duijn, C.M., Oostra, B.A. \& Heutink, P. (2003). Mutations in the DJ-1 gene associated with autosomal recessive early-onset parkinsonism. Science, 299, pp. 256-9.

Botella, J.A.; Bayersdorfer, F. \& Schneuwly, S. (2008). Superoxide dismutase overexpression protects dopaminergic neurons in a Drosophila model of Parkinson's disease. Neurobiol Dis, 30, pp. 65-73.

Braak, H.; Del Tredici, K., Rüb, U., de Vos, R.A., Jansen Steur, E.N. \& Braak, E. (2003). Staging of brain pathology related to sporadic Parkinson's disease. Neurobiol Aging, 24, pp. 197-211.

Brand, A.H.; \& Perrimon, N. (1993). Targeted gene expression as a means of altering cell fates and generating dominant phenotypes. Development, 118, pp. 401-15.

Cha G.H.; Kim, S., Park, J., Lee, E., Kim, M., Lee, S.B., Kim, J.M., Chung, J. \& Cho K. S. (2005), Parkin negatively regulates JNK pathway in the dopaminergic neurons of Drosophila. Proc Natl Acad Sci U S A, 102, pp. 10345-50.

Chen, H.; \& Chan, D.C. (2009), Mitochondrial dynamics--fusion, fission, movement, and mitophagy-in neurodegenerative diseases. Hum Mol Genet, 18, pp. R169-76.

Chen, L.; \& Feany, M.B. (2005). Alpha-synuclein phosphorylation controls neurotoxicity and inclusion formation in a Drosophila model of Parkinson disease. Nat Neurosci, 8, pp. 657-63.

Clark, I.E.; Dodson, M.W., Jiang, C., Cao, J.H., Huh, J.R., Seol, J.H., Yoo, S.J., Hay, B.A. \& Guo, M. (2006). Drosophila pink1 is required for mitochondrial function and interacts genetically with parkin. Nature, 441, pp. 1162-6.

Cooper, A.A.; Gitler, A.D., Cashikar, A., Haynes, C.M., Hill, K.J., Bhullar, B., Liu, K., Xu, K., Strathearn, K.E., Liu, F., Cao, S., Caldwell, K.A., Caldwell, G.A., Marsischky, G., Kolodner, R.D., Labaer, J., Rochet, J.C., Bonini, N.M. \& Lindquist, S. (2006). Alphasynuclein blocks ER-Golgi traffic and Rab1 rescues neuron loss in Parkinson's models. Science, 313, pp. 324-8.

Coulom H.; \& Birman S. (2004). Chronic exposure to rotenone models sporadic Parkinson's disease in Drosophila melanogaster. J Neurosci, 24, pp. 10993-8.

Darios, F.; Corti, O., Lücking, C.B., Hampe, C., Muriel, M.P., Abbas, N., Gu, W.J., Hirsch, E.C., Rooney, T., Ruberg, M., \& Brice, A. (2003). Parkin prevents mitochondrial swelling and cytochrome c release in mitochondria-dependent cell death. Hum Mol Genet, 12, pp. 517-26.

Dauer, W.; \& Przedborski, S. (2003). Parkinson's disease: mechanisms and models. Neuron, 39, pp. 889-909. 
Deng, H.; Dodson, M.W., Huang, H. \& Guo, M. (2008). The Parkinson's disease genes pink1 and parkin promote mitochondrial fission and/or inhibit fusion in Drosophila. Proc Natl Acad Sci U S A, 105, pp. 14503-8.

Feany, M.B.; \& Bender, W.W. (2000). A Drosophila model of Parkinson's disease. Nature, 404, pp. 394-8.

Fujiwara, H.; Hasegawa, M., Dohmae, N., Kawashima, A., Masliah, E., Goldberg, M.S., Shen, J., Takio, K. \& Iwatsubo, T. (2002). alpha-Synuclein is phosphorylated in synucleinopathy lesions. Nat Cell Biol, 4, pp. 160-4.

Geisler, S.; Holmström, K.M., Skujat, D., Fiesel, F.C., Rothfuss, O.C., Kahle, P.J. \& Springer W. (2010). PINK1/Parkin-mediated mitophagy is dependent on VDAC1 and p62/SQSTM1. Nat Cell Biol, 12, pp. 119-31.

Goldman, S.J.; Taylor, R., Zhang, Y. \& Jin, S. (2010). Autophagy and the degradation of mitochondria. Mitochondrion, 10, pp. 309-15.

Greene, J.C.; Whitworth, A.J., Kuo, I., Andrews, L.A., Feany, M.B. \& Pallanck, L.J. (2003). Mitochondrial pathology and apoptotic muscle degeneration in Drosophila parkin mutants. Proc Natl Acad Sci U S A, 100, pp. 4078-83.

Greene, J.C.; Whitworth, A.J., Andrews, L.A., Parker, T.J. \& Pallanck, L.J. (2005). Genetic and genomic studies of Drosophila parkin mutants implicate oxidative stress and innate immune responses in pathogenesis. Hum Mol Genet, 14, pp. 799-811.

Hardy, J.; Lewis, P., Revesz, T., Lees, A. \& Paisan-Ruiz, C. (2009). The genetics of Parkinson's syndromes: a critical review. Curr Opin Genet Dev, 19, pp. 254-65.

Haywood, A.F.; \& Staveley, B.E. (2004). Parkin counteracts symptoms in a Drosophila model of Parkinson's disease. BMC Neurosci, 5:14.

Haywood, A.F.; \& Staveley, B.E. (2006). Mutant alpha-synuclein-induced degeneration is reduced by parkin in a fly model of Parkinson's disease. Genome, 49, pp. 505-10.

Horowitz, J.M.; Vernace, V.A., Myers, J., Stachowiak, M.K., Hanlon, D.W., Fraley, G.S. \& Torres, G. (2001). Immunodetection of Parkin protein in vertebrate and invertebrate brains: a comparative study using specific antibodies. J Chem Neuroanat, 21, pp. 7593.

Imai, Y.; Gehrke, S., Wang, H.Q., Takahashi, R., Hasegawa, K., Oota, E. \& Lu B. (2008). Phosphorylation of $4 \mathrm{E}-\mathrm{BP}$ by LRRK2 affects the maintenance of dopaminergic neurons in Drosophila. EMBO J, 27, pp. 2432-43.

Kanao, T.; Venderova, K., Park, D.S., Unterman, T., Lu, B. \& Imai Y. (2010). Activation of FoxO by LRRK2 induces expression of proapoptotic proteins and alters survival of postmitotic dopaminergic neuron in Drosophila. Hum Mol Genet, 19, pp. 374758.

Kim, Y.; Park, J., Kim, S., Song, S., Kwon, S.K., Lee, S.H., Kitada, T., Kim, J.M. \& Chung, J. (2008). PINK1 controls mitochondrial localization of Parkin through direct phosphorylation. Biochem Biophys Res Commun, 377, pp. 975-80.

Kitada, T.; Asakawa, S., Hattori, N., Matsumine, H., Yamamura, Y., Minoshima, S., Yokochi, M., Mizuno, Y. \& Shimizu N. (1998). Mutations in the parkin gene cause autosomal recessive juvenile parkinsonism. Nature, 392, pp. 605-8. 
Kramer, J.M.; \& Staveley, B.E. (2003). GAL4 causes developmental defects and apoptosis when expressed in the developing eye of Drosophila melanogaster. Genet Mol Res, 2, pp. 43-7.

Krüger R.; Kuhn, W., Müller, T., Woitalla, D., Graeber, M., Kösel, S., Przuntek, H., Epplen, J.T., Schöls, L. \& Riess, O. (1998). Ala30Pro mutation in the gene encoding alphasynuclein in Parkinson's disease. Nat Genet, 18, pp. 106-8.

Lee, S.B.; Kim, W., Lee, S. \& Chung J. (2007). Loss of LRRK2/PARK8 induces degeneration of dopaminergic neurons in Drosophila. Biochem Biophys Res Commun, 358, pp. 5349.

Liu, Z.; Wang, X., Yu, Y., Li, X., Wang, T., Jiang, H., Ren, Q., Jiao, Y., Sawa, A., Moran, T., Ross, C.A., Montell, C. \& Smith, W.W. (2008). A Drosophila model for LRRK2linked parkinsonism. Proc Natl Acad Sci U S A, 105, pp. 2693-8.

Menzies, F.M.; Yenisetti, S.C. \& Min, K.T. (2005). Roles of Drosophila DJ-1 in survival of dopaminergic neurons and oxidative stress. Curr Biol, 15, 1578-82.

Meulener, M.; Whitworth, A.J., Armstrong-Gold, C.E., Rizzu, P., Heutink, P., Wes, P.D., Pallanck, L.J. \& Bonini, N.M. (2005). Drosophila DJ-1 mutants are selectively sensitive to environmental toxins associated with Parkinson's disease. Curr Biol, 15, pp. 1572-7.

Meulener, M.C.; Xu, K., Thomson. L., Ischiropoulos, H. \& Bonini, N.M. (2006). Mutational analysis of DJ-1 in Drosophila implicates functional inactivation by oxidative damage and aging. Proc Natl Acad Sci U S A, 103, pp. 12517-22.

Narendra, D.P.; Jin, S.M., Tanaka, A., Suen, D.F., Gautier, C.A., Shen, J., Cookson, M.R. \& Youle, R.J. (2010). PINK1 is selectively stabilized on impaired mitochondria to activate Parkin. PLoS Biol, 8, pp. e1000298.

Nässel, D.R.; \& Elekes, K. (1992). Aminergic neurons in the brain of blowflies and Drosophila: dopamine- and tyrosine hydroxylase-immunoreactive neurons and their relationship with putative histaminergic neurons. Cell Tissue Res, 267, pp. 14767.

Paisán-Ruíz, C.; Jain, S., Evans, E.W., Gilks, W.P., Simón, J., van der Brug, M., López de Munain, A., Aparicio, S., Gil, A.M., Khan, N., Johnson, J., Martinez, J.R., Nicholl, D., Carrera, I.M., Pena, A.S., de Silva, R., Lees, A., Martí-Massó, J.F., Pérez-Tur, J. \& Wood, N.W. \& Singleton, A.B. (2004). Cloning of the gene containing mutations that cause PARK8-linked Parkinson's disease. Neuron, 44, pp. 595-600.

Park, J.; Lee, S.B., Lee, S., Kim, Y., Song, S., Kim, S., Bae, E., Kim, J., Shong, M., Kim, J.M. \& Chung, J. (2006). Mitochondrial dysfunction in Drosophila PINK1 mutants is complemented by parkin. Nature, 441, pp. 1157-61.

Park, J.; Lee, G. \& Chung, J. (2009). The PINK1-Parkin pathway is involved in the regulation of mitochondrial remodeling process. Biochem Biophys Res Commun, 378, pp. 518-23.

Park, S.S.; Schulz, E.M. \& Lee, D. (2007). Disruption of dopamine homeostasis underlies selective neurodegeneration mediated by alpha-synuclein. Eur J Neurosci, 26, pp. 3104-12. 
Pendleton, R.G.; Parvez, F., Sayed, M. \& Hillman R. (2002). Effects of pharmacological agents upon a transgenic model of Parkinson's disease in Drosophila melanogaster. J Pharmacol Exp Ther, 300, pp. 91-6.

Pesah, Y.; Pham, T., Burgess, H., Middlebrooks, B., Verstreken, P., Zhou, Y., Harding, M., Bellen, H. \& Mardon G. (2004). Drosophila parkin mutants have decreased mass and cell size and increased sensitivity to oxygen radical stress. Development, 131, pp. 2183-94.

Polymeropoulos, M.H.; Lavedan, C., Leroy, E., Ide, S.E., Dehejia, A., Dutra, A., Pike, B., Root, H., Rubenstein, J., Boyer, R., Stenroos, E.S., Chandrasekharappa, S., Athanassiadou, A., Papapetropoulos, T., Johnson, W.G., Lazzarini, A.M., Duvoisin, R.C., Di Iorio, G., Golbe, L.I. \& Nussbaum, R.L. (1997). Mutation in the alphasynuclein gene identified in families with Parkinson's disease. Science, 276, pp. 2045-7.

Poole, A.C.; Thomas, R.E., Andrews, L.A., McBride, H.M., Whitworth, A.J. \& Pallanck, L.J. (2008). The PINK1/Parkin pathway regulates mitochondrial morphology. Proc Natl Acad Sci U S A, 105, pp. 1638-43.

Rezával, C.; Werbajh, S. \& Ceriani, M.F. (2007). Neuronal death in Drosophila triggered by GAL4 accumulation. Eur J Neurosci, 25, pp. 683-94.

Riparbelli, M.G.; \& Callaini, G. (2007). The Drosophila parkin homologue is required for normal mitochondrial dynamics during spermiogenesis. Dev Biol, 303, pp. 108-20.

Sang, T.K.; Chang, H.Y., Lawless, G.M., Ratnaparkhi, A., Mee, L., Ackerson, L.C., Maidment, N.T., Krantz, D.E. \& Jackson, G.R. (2007). A Drosophila model of mutant human parkin-induced toxicity demonstrates selective loss of dopaminergic neurons and dependence on cellular dopamine. J Neurosci, 27, pp. 981-92.

Simuni, T.; \& Sethi, K. (2008). Nonmotor manifestations of Parkinson's disease. Ann Neurol, 64, pp. S65-80.

Singleton, A.B.; Farrer, M., Johnson, J., Singleton, A., Hague, S., Kachergus, J., Hulihan, M., Peuralinna, T., Dutra, A., Nussbaum, R., Lincoln, S., Crawley, A., Hanson, M., Maraganore, D., Adler, C., Cookson, M.R., Muenter, M., Baptista, M., Miller, D., Blancato, J., Hardy, J., \& Gwinn-Hardy, K. (2003). alpha-Synuclein locus triplication causes Parkinson's disease. Science, 302, pp. 841.

Todd, A.M.; \& Staveley, B.E. (2008). Pink1 suppresses alpha-synuclein-induced phenotypes in a Drosophila model of Parkinson's disease. Genome, 51, pp. 1040-6.

Trinh, K.; Moore, K., Wes, P.D., Muchowski, P.J., Dey, J., Andrews, L. \& Pallanck LJ. (2008). Induction of the phase II detoxification pathway suppresses neuron loss in Drosophila models of Parkinson's disease. J Neurosci, 28, pp. 465-72.

Valente, E.M.; Abou-Sleiman, P.M., Caputo, V., Muqit, M.M., Harvey, K., Gispert, S., Ali, Z., Del Turco, D., Bentivoglio, A.R., Healy, D.G., Albanese, A., Nussbaum, R., González-Maldonado, R., Deller, T., Salvi, S., Cortelli, P., Gilks, W.P., Latchman, D.S., Harvey, R.J., Dallapiccola, B., Auburger, G. \& Wood N.W. (2004). Hereditary early-onset Parkinson's disease caused by mutations in PINK1. Science, 304, pp. 1158-60. 
Vives-Bauza, C.; Zhou, C., Huang, Y., Cui, M., de Vries, R.L., Kim, J., May, J., Tocilescu, M.A., Liu, W., Ko, H.S., Magrané, J., Moore, D.J., Dawson, V.L., Grailhe, R., Dawson, T.M., Li, C., Tieu, K. \& Przedborski, S. (2010). PINK1-dependent recruitment of Parkin to mitochondria in mitophagy. Proc Natl Acad Sci U S A, 107, pp. 378-83.

Wang, D.; Qian, L., Xiong, H., Liu, J., Neckameyer, W.S., Oldham, S., Xia, K., Wang, J., Bodmer, R.\& Zhang, Z. (2006). Antioxidants protect PINK1-dependent dopaminergic neurons in Drosophila. Proc Natl Acad Sci U S A, 103, pp. 13520-5.

Wang, C.; Lu, R., Ouyang, X., Ho, M.W., Chia, W., Yu, F. \& Lim, K.L. (2007). Drosophila overexpressing parkin R275W mutant exhibits dopaminergic neuron degeneration and mitochondrial abnormalities. J Neurosci, 27,pp. 8563-70.

Wassef, R.; Haenold, R., Hansel, A., Brot, N., Heinemann, S.H. \& Hoshi, T. (2007). Methionine sulfoxide reductase A and a dietary supplement S-methyl-L-cysteine prevent Parkinson's-like symptoms. J Neurosci, 27, pp. 12808-16.

Whitworth, A.J.; Theodore, D.A., Greene, J.C., Benes, H., Wes, P.D. \& Pallanck, L.J. (2005). Increased glutathione S-transferase activity rescues dopaminergic neuron loss in a Drosophila model of Parkinson's disease. Proc Natl Acad Sci U S A, 102, pp. 8024-9.

Whitworth, A.J.; Wes, P.D. \& Pallanck, L.J. (2006). Drosophila models pioneer a new approach to drug discovery for Parkinson's disease. Drug Discov Today, 11, pp. 11926.

Whitworth, A.J.; Lee, J.R., Ho, V.M., Flick, R., Chowdhury, R. \& McQuibban, G.A. (2008). Rhomboid-7 and HtrA2/Omi act in a common pathway with the Parkinson's disease factors Pink1 and Parkin. Dis Model Mech, 1, pp. 168-74.

Xiong, H.; Wang, D., Chen, L., Choo, Y.S., Ma, H., Tang, C., Xia, K., Jiang, W., Ronai, Z., Zhuang, X. \& Zhang, Z. (2009). Parkin, PINK1, and DJ-1 form a ubiquitin E3 ligase complex promoting unfolded protein degradation. J Clin Invest, 119, pp. 650-60.

Yang, Y.; Nishimura, I., Imai, Y., Takahashi, R. \& Lu, B. (2003). Parkin suppresses dopaminergic neuron-selective neurotoxicity induced by Pael-R in Drosophila. Neuron, 37, pp. 911-24.

Yang, Y.; Gehrke, S., Imai, Y., Huang, Z., Ouyang, Y., Wang, J.W., Yang, L., Beal, M.F., Vogel, H. \& Lu, B. (2006). Mitochondrial pathology and muscle and dopaminergic neuron degeneration caused by inactivation of Drosophila Pink1 is rescued by Parkin. Proc Natl Acad Sci U S A, 103, pp. 10793-8.

Yang, Y.; Ouyang, Y., Yang, L., Beal, M.F., McQuibban, A., Vogel, H. \& Lu, B. (2008). Pink1 regulates mitochondrial dynamics through interaction with the fission/fusion machinery. Proc Natl Acad Sci U S A, 105, pp. 7070-5.

Zarranz, J.J.; Alegre, J., Gómez-Esteban, J.C., Lezcano, E., Ros, R., Ampuero, I., Vidal, L., Hoenicka, J., Rodriguez, O., Atarés, B., Llorens, V., Gomez Tortosa, E., del Ser, T., Muñoz, D.G. \& de Yebenes, J.G. (2004). The new mutation, E46K, of alphasynuclein causes Parkinson and Lewy body dementia. Ann Neurol, 55, pp. 16473. 
Zhou, C.; Huang, Y., Shao, Y., May, J., Prou, D., Perier, C., Dauer, W., Schon, E.A. \& Przedborski, S. (2008). The kinase domain of mitochondrial PINK1 faces the cytoplasm. Proc Natl Acad Sci U S A, 105, pp. 12022-7.

Zimprich, A.; Biskup, S., Leitner, P., Lichtner, P., Farrer, M., Lincoln, S., Kachergus, J., Hulihan, M., Uitti, R.J., Calne, D.B., Stoessl, A.J., Pfeiffer, R.F., Patenge, N., Carbajal, I.C., Vieregge, P., Asmus, F., Müller-Myhsok, B., Dickson, D.W., Meitinger, T., Strom, T.M., Wszolek, Z.K. \& Gasser, T. (2004). Mutations in LRRK2 cause autosomal-dominant parkinsonism with pleomorphic pathology. Neuron, 44, pp. 601-7.

Ziviani, E; Tao, R.N. \& Whitworth, A.J. (2010). Drosophila parkin requires PINK1 for mitochondrial translocation and ubiquitinates mitofusin. Proc Natl Acad Sci U S A, 107, pp. 5018-23. 


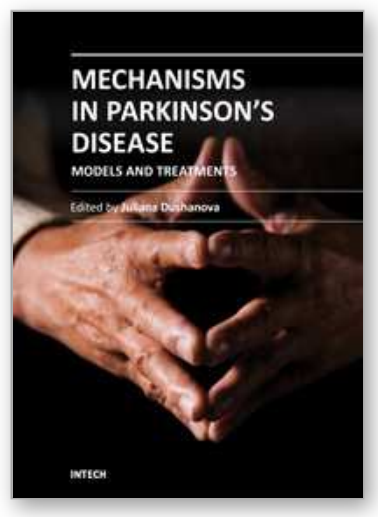

\author{
Mechanisms in Parkinson's Disease - Models and Treatments \\ Edited by Dr. Juliana Dushanova
}

ISBN 978-953-307-876-2

Hard cover, 582 pages

Publisher InTech

Published online 08, February, 2012

Published in print edition February, 2012

Parkinson's disease (PD) results primarily from the death of dopaminergic neurons in the substantia nigra. Current PD medications treat symptoms; none halt or retard dopaminergic neuron degeneration. The main obstacle to developing neuroprotective therapies is a limited understanding of the key molecular mechanisms that provoke neurodegeneration. The discovery of PD genes has led to the hypothesis that misfolding of proteins and dysfunction of the ubiquitin-proteasome pathway are pivotal to PD pathogenesis. Previously implicated culprits in PD neurodegeneration, mitochondrial dysfunction, and oxidative stress may also act in part by causing the accumulation of misfolded proteins, in addition to producing other deleterious events in dopaminergic neurons. Neurotoxin-based models have been important in elucidating the molecular cascade of cell death in dopaminergic neurons. PD models based on the manipulation of PD genes should prove valuable in elucidating important aspects of the disease, such as selective vulnerability of substantia nigra dopaminergic neurons to the degenerative process.

\title{
How to reference
}

In order to correctly reference this scholarly work, feel free to copy and paste the following:

Brian E. Staveley (2012). Successes of Modelling Parkinson Disease in Drosophila, Mechanisms in Parkinson's Disease - Models and Treatments, Dr. Juliana Dushanova (Ed.), ISBN: 978-953-307-876-2, InTech, Available from: http://www.intechopen.com/books/mechanisms-in-parkinson-s-disease-models-andtreatments/successes-of-modelling-parkinson-disease-in-drosophila-

\section{INTECH}

open science | open minds

\section{InTech Europe}

University Campus STeP Ri

Slavka Krautzeka 83/A

51000 Rijeka, Croatia

Phone: +385 (51) 770447

Fax: +385 (51) 686166

www.intechopen.com

\section{InTech China}

Unit 405, Office Block, Hotel Equatorial Shanghai

No.65, Yan An Road (West), Shanghai, 200040, China

中国上海市延安西路65号上海国际贵都大饭店办公楼405单元

Phone: +86-21-62489820

Fax: $+86-21-62489821$ 
(C) 2012 The Author(s). Licensee IntechOpen. This is an open access article distributed under the terms of the Creative Commons Attribution 3.0 License, which permits unrestricted use, distribution, and reproduction in any medium, provided the original work is properly cited. 